\title{
Hypothyroidism in hemi-thyroidectomy cases
}

\begin{abstract}
Introduction: Hemithyroidectomy is usually preferred when single non-malignant nodule is there. In many instances hemithyroidectomy is conducted in cases with suspicious lesion for diagnosis and to avoid unnecessary total thyroidectomy assuming that this many preserve a normal thyroid function post operatively.
\end{abstract}

Method: A prospective chart review study. We reviewed the records of 88 euthyroid patients who underwent hemithyroidectomy and followed up their thyroid function post-operatively to evaluate the incidence of hypothyroidism in these cases.

Results: Out of 88 patients, 48 patients met the inclusion criteria. 11 males, 37 females. $56.3 \%$ of those who underwent hemithyroidectomy developed hypothyroidism within the next 6 months and continue to need thyroxin supplementation up to date. This risk found to be relatively higher in females $(60 \%)$, young age group (66\%), and those with thyroiditis $(100 \%)$. Transient hypothyroidism occurs in $4.16 \%$ only, where thyroid function returned to normal within 6 months postoperatively.

Conclusion: Hypothyroidism occurs in more than half of hemithyroidectomy cases and was transient in the minority of them only. Doing total thyroidectomy in cases of risky recurrence or remnants, however other complications to be considered can be better than hemithyroidectomy.
Volume 10 Issue 2 - 2018

Ibrahim Sumaily,' Majed Assiri, ${ }^{2}$ Mohammad Alshareef, ${ }^{2}$ Ramzi Daghriri, ${ }^{2}$ Saad Alqarni ${ }^{2}$ 'ORL-HNS Department, King Fahd Central Hospital, Jazan, Saudi Arabia

${ }^{2}$ ORL-HNS Department, Asir Central Hospital, Saudi Arabia

Correspondence: Ibrahim Sumaily, ORL-HNS

Department, King Fahd Central Hospital, KSA, Jazan, Saudi Arabia, Tel +966504621902,

Email sumaily.i@gmail.com

Received: February 23, 2018 | Published: April 03, 2018

\section{Introduction and aim}

Usually total thyroidectomy, not like hemithyroidectomy, leads to a lifelong thyroxin supplementation. With some thyroid diseases doing hemithyroidectomy may carry a risk for recurrence or remnants. The complications of hemithyroidectomy surgery including bleeding, infection, recurrent and superior laryngeal nerves injury and hypoparathyroidism are much less than the incidence of hypothyroidism, which is ranging between 5 to $49 \%$ in comparison to other complications which represent about $1 \%$. Knowing this and the risk of having residual or recurrent disease can be encouraging to the surgeon to proceed to total thyroidectomy. In our region, southern region of Saudi Arabia, no study tackled the incidence of hypothyroidism in hemithyroidectomy cases. Herein we studied this entity, so if it usually ends with hypothyroidism then total thyroidectomy may be considered to be the procedure of choice in these cases.

\section{Method}

A prospective charts review study. We reviewed the records of 88 patients who underwent hemithyroidectomy between 2012-2016. Inclusion Criteria: Hemithyroidectomy, normal preoperative thyroid function tests (TFT) and not on thyroxin preoperatively. Exclusion Criteria: All patients who underwent completion surgery, preoperative hypothyroidism even if it worsens postoperatively, those with malignant disease and those who have no incomplete records.

Limitations: Small sample number, lack of follow up of some cases, and unknown exact amount of thyroid tissue being removed and wither the difference in this amount is a risk or not.
The pre-operative TFT obtained within 2 weeks of surgery and post operative TFT obtained 1 month after surgery, then 3 months later, then 6 months later. The results of TFT were studied for acute and persistent hypothyroidism in these patients. SPSS v.22 used for data analysis.

\section{Results}

Out of 88 cases, 48 met our inclusion criteria. 11 males, 37 females. Mean age was 36.5 years old. The candidates divided into 3 groups of age: $6 \%$ less 20 years old, $79 \%$ between 20 and 50 years old and $14.5 \%$ elder than 50 years old. 22 operated by GS, and 26 by ENT.

Within the first one month, $60.4 \%$ of the cases developed hypothyroidism. Of them, 2 patients (7.5\%) return to normal thyroid function within six months post operatively. By six months, a total of 27 out of 48 patients $(56.3 \%)$ were hypothyroid. None of these patients' thyroid status improved later on. The other $43.8 \%$ found to be satisfied with their gland's remnants without any need for supplementation.

The risk of developing persistent hypothyroidism in hemithyroidectomy was significantly higher in females in comparison to males, $62.2 \%$ and $36.4 \%$ respectively. $8.3 \%$ of the cases found to have picture of thyroiditis in histopathology sample. All of them developed persistent hypothyroidism $(14.8 \%$ of hypothyroidism group).

The risk of hypothyroidism in hemithyroidectomy was relatively higher in young and those elder than 50 (66\% and 57\% respectively). Transient hypothyroidism found to occur in $4.16 \%$ middle age females only. Youngs and elderly and male gender showed no improvement later on. None of those who were euthyroid postoperatively developed 
hypothyroidism up to date. No significant difference in this outcome between those who were operated by ENT or by GS.

\begin{tabular}{lll|r|r|r|}
\hline & & \multicolumn{2}{|c|}{ one month TFT results } & \multirow{2}{*}{ Total } \\
\cline { 3 - 4 } & & \multicolumn{1}{|c|}{ Normal } & decreased & \multicolumn{1}{c|}{11} \\
\hline patient gender & male & Count & 7 & 4 & 11 \\
& & \% within patient gender & $63.6 \%$ & $36.4 \%$ & $100.0 \%$ \\
\cline { 2 - 5 } & female & Count & 12 & 25 & 37 \\
& & \% within patient gender & $32.4 \%$ & $67.6 \%$ & $100.0 \%$ \\
\hline Total & Count & 19 & 29 & 48 \\
& \% within patient gender & $39.6 \%$ & $60.4 \%$ & $100.0 \%$ \\
\hline
\end{tabular}

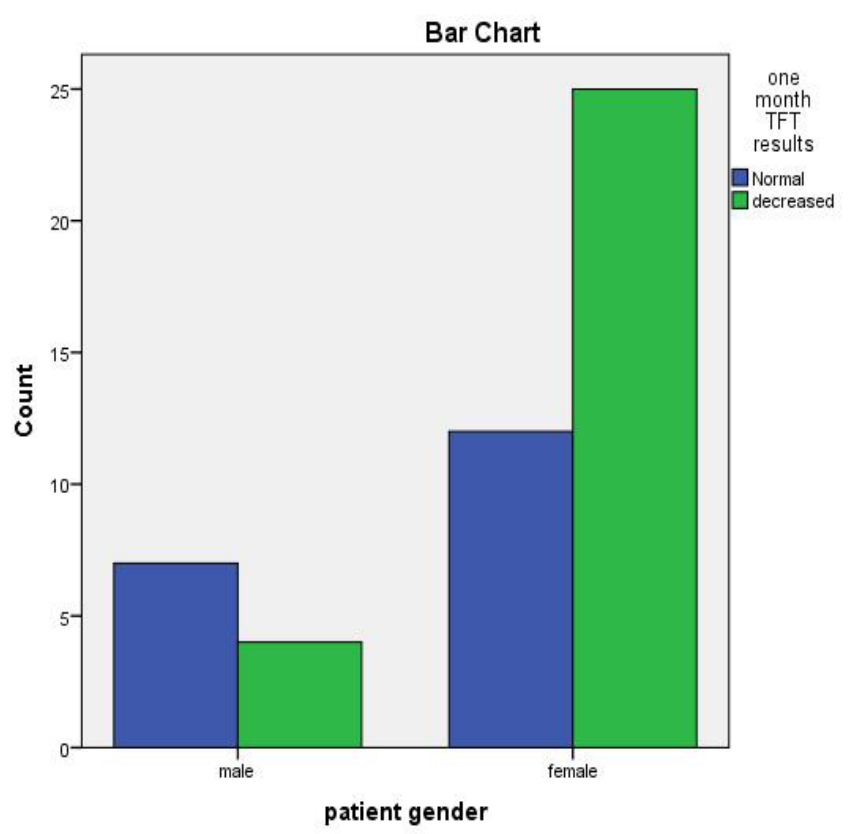

\section{Discussion}

Hemithyroidectomy is usually used in cases of single benign nodule and for diagnosis in cases where the fine needle aspiration was not enough to rule out malignancy. It may be preferred by the surgeon when it is possible to avoid the possible sequalae of total thyroidectomy including hypothyroidism, hypoparathyroidism and laryngeal nerves injury. Although the literature is rich in thyroid studies involving our topic, it is not will studied among our country, and no similar study in our region, southern region of Saudi Arabia. The incidence of hypothyroidism is higher in our study $(56.3 \%)$ in comparison to previous studies, which showed an average of about $22 \% .^{1}$ In our study; young age is relatively a risk factor for hypothyroidism in hemithyroidectomy. Several studies showed that the age is a risk factor. ${ }^{1,45}$ But more studies showed that there is no relation between age and the risk of hypothyroidism in hemithyroidectomy. ${ }^{1-3,6-9}$ Female gender noticed to be a risk factor developing hypothyroidism in hemithyroidectomy. There is a chance of transient hypothyroidism in hemithyroidectomy. ${ }^{2}$ The timing of diagnosis is variable among the studies. Most often hypothyroidism diagnosed in the first 3 months after hemithyroidectomy. ${ }^{10}$ In our study all the cases that developed hypothyroidism were diagnosed in the first one month. The improvement usually occurs within few months. ${ }^{3}$ In our study it was $4.16 \%$ who improved within six months. All of them fall in the second group of age.

\section{Conclusion}

Hypothyroidism is a common complication of hemithyroidectomy occurring in more than half of the cases. Transient Hypothyroidism can be the condition in minority of the cases. However other complications to be considered, total thyroidectomy in cases with high risk of recurrence or remnants, young age group and females, may be better than doing hemithyroidectomy. Further study is recommended to weigh benefits and risks of doing total thyroidectomy in those who basically are planned for hemithyroidectomy.

\section{Acknowledgment}

None.

\section{Conflict of interest}

None.

\section{References}

1. Verloop H, Louwerens M, Schoones JW, et al. Risk of hypothyroidism following hemithyroidectomy: systematic review and meta-analysis of prognostic studies. J Clin Endocrinol Metab. 2012;97(7):2243-2255.

2. Piper HG, Bugis SP, Wilkins GE, et al. Detecting and defining hypothyroidism after hemithyroidectomy. Am J Surg 2005;189(5):587591.

3. Barsano CP. Other forms of primary hypothyroidism. In: Braverman LE, editor. WERNER and INGBAR's the thyroid. 6th ed. New York: Lippincott; 1991:959-960.

4. Beisa V, Kazanavicius D, Skrebunas A, et al. Prognosis of thyroid function after hemithyroidectomy. Central European Journal of Medicine. 2010;6(2):152-157.

5. Lindblom P, Valdemarsson S, Lindergård B, et al. Decreased levels of ionized calcium one year after hemi-thyroidectomy: importance of reduced thyroid hormones. Horm Res. 2001;55(2):81-87.

6. McHenry CR, Slusarczyk SJ. Hypothyroidism following hemithyroidectomy: incidence, risk factors, and management. Surgery. 2000;128(6):994-998.

7. Seiberling KA, Dutra JC, Bajaramovic S. Hypothyroidism fol-lowing hemithyroidectomy for benign nontoxic thyroid disease. Ear Nose Throat J. 2007;86(5):295-299.

8. Koh YW, Lee SW, Choi EC, et al. Prediction of hypothyroidism after hemithyroid-ectomy: a biochemical and pathological analysis. Eur Arch Otorhi-nolaryngol. 2008;265(4):453-457.

9. Stoll SJ, Pitt SC, Liu J, et al. Thyroid hormone replacement after thyroid lobectomy. Surgery. 2009;146(4):554-558.

10. Ahn D, Sohn JH1, Jeon JH. Hypothyroidism following hemithyroidectomy: incidence, risk factors, and clinical characteristics. J Clin Endocrinol Metab. 2016;101(4):1429-1436. 